\title{
Novel Approaches for Exclusive and Continuous Fingerprint Classification
}

\author{
Javier A. Montoya-Zegarra ${ }^{1,2}$, João P. Papa ${ }^{2}$, Neucimar J. Leite ${ }^{2}$, \\ Ricardo da Silva Torres ${ }^{2}$, and Alexandre X. Falcão ${ }^{2}$ \\ ${ }^{1}$ Computer Engineering Department, Faculty of Engineering, San Pablo Catholic University, \\ Av. Salaverry 301, Vallecito, Arequipa, Peru \\ ${ }^{2}$ Institute of Computing, State University of Campinas, \\ Av. Albert Einstein 1216, Campinas, São Paulo, Brazil \\ \{jmontoyaz, papa.joaopaulo\}@gmail.com
}

\begin{abstract}
This paper proposes novel exclusive and continuous approaches to guide the search and the retrieval in fingerprint image databases. Both approaches are useful to perform a coarse level classification of fingerprint images before fingerprint authentication tasks. Our approaches are characterized by: (1) texture image descriptors based on pairs of multi-resolution decomposition methods that encode effectively global and local fingerprint information, with similarity measures used for fingerprint matching purposes, and (2) a novel multi-class object recognition method based on the Optimum Path Forest classifier. Experiments were carried out on the standard NIST-4 dataset aiming to study the discriminative and scalability capabilities of our approaches. The high classification rates allow us demonstrate the feasibility and validity of our approaches for characterizing fingerprint images accurately.
\end{abstract}

\section{Introduction}

Fingerprints are considered nowadays one of the most reliable biometric characteristic for human recognition due to their individuality and persistence [1]. Traditionally, a fingerprint-recognition system may operate in two distinct modes [2]: verification and identification. In verification mode, the system determines whether an input fingerprint corresponds to a claimed identity by performing one-to-one comparisons. Because of the discriminating power needs for establishing the subjects identity, local fingerprint information such as minutiae and, more recently, pores are used for that purposes (they are believed to be unique across individuals and across fingers of the same individual) [3]. In identification mode, the system identifies which database images resemble best an input fingerprint image without having any kind of a-priori knowledge. To accomplish this task, the system performs one-to-many comparisons and uses typically the global information of fingerprints contained in their central region (ridge and valley structures).

While systems operating in verification mode establish the identity of an individual, systems in identification mode are useful to reduce search spaces during the matching phase and therefore play an important role during the recognition process. Despite the concerted efforts of researchers in fingerprint identification, this objective remains

T. Wada, F. Huang, and S. Lin (Eds.): PSIVT 2009, LNCS 5414, pp. 386-397, 2009.

(C) Springer-Verlag Berlin Heidelberg 2009 
open [4]. In fact, the popularity of fingerprint-based recognition has led to large-scale databases. While the large size of these collections compromise the retrieval speed, the presence of noise and distortions in fingerprint images may reduce the overall retrieval accuracy. Therefore, both retrieval accuracy and speed are key factors during the fingerprint recognition process.

Roughly speaking, there are two kinds of approaches that can be used to reduce the retrieval space during fingerprint matching, they are namely the exclusive-, and the continuous-fingerprint classification [5]. The former uses some high-level characteristics to partitionate the fingerprint database into mutual exclusive bins. Once the fingerprint query image is classified, it will be searched only in its corresponding bin. In the latter, the fingerprint images are not represented by a single class, but by feature vectors spread over a feature space. Hence, database images that fall within a given radius to the query fingerprints are considered as valid matches. The objective of both approaches is to characterize the fingerprint images by some global information for indexing purposes, instead of offering some kind of discriminatory information for recognition.

In this context, we propose novel exclusive and continuous approaches to guide the search and the retrieval in fingerprint image databases. More specifically, they are used to perform a coarse level classification of fingerprint images before fingerprint recognition tasks. Our approaches are characterized by: (1) texture image descriptors based on multi-resolution decomposition methods that encode effectively global and local fingerprint information, and (2) a novel multi-class object recognition method based on the Optimum Path Forest classifier [6], which models the pattern recognition problem as a optimum partition of the feature space according to some criterion optimality. Since in identification tasks, the similarity measures play also an important role, we show how, in combination, with our fingerprint representation methods, the accuracy of our systems is improved.

The outline of this paper is as follows. In the next section, we introduce the architecture of our system, whilst Section 3 describes how the fingerprint images are represented as feature vectors. Section 4 introduces the Optimum Path Forest classifier method, which is the basis of our exclusive classification method. The experimental setup conducted in our study is presented in Section 5. In section 6, experimental results on the standard Nist 4 databse are given and are used to show the high accuracy of our approaches. Finally, some conclusions are drawn in Section 7 .

\section{System Overview}

The architecture of our proposed approach, presented in Figure 1 can be divided into two main subsystems, namely, the enrollment- and the query-subsystem. The enrollmentsubsystem acquires the information that will be stored in the database for later use. On the other side, the query subsystem is responsible for retrieving similar fingerprints from the database according to the user's fingerprint query image. Our system operates as follows:

1. Enrollment-subsystem: several fingerprint images are first captured (arrow labeled 1 in Figure 1) and a Region of Interest (ROI) within the fingerprint is marked (module 1 , arrow 2) by a center point area detection module. The fingerprint ROI is represented by the central part of the fingerprint images, since most of the category 


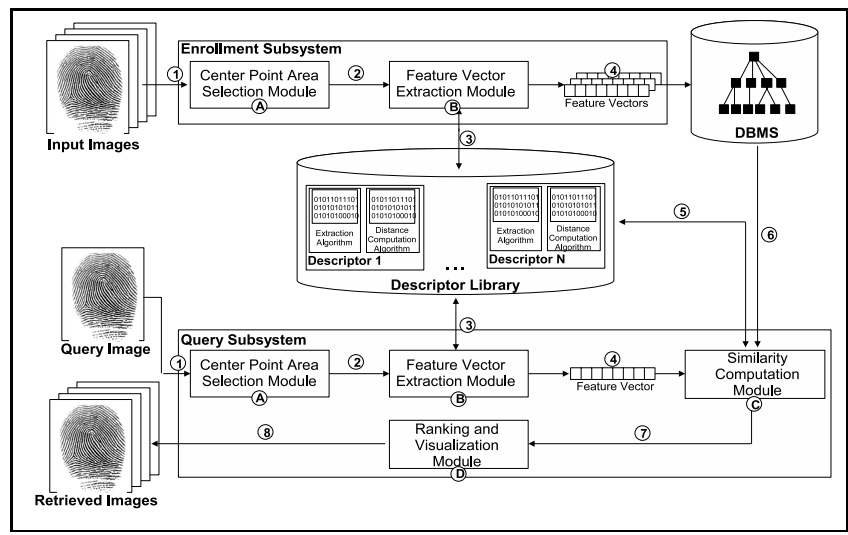

Fig. 1. Architecture of the proposed system

information is contained in it. The feature extraction algorithms contained in the descriptor library (module B, arrow 3 ) are used by the feature extraction module to generate the features (arrow 4) that are stored for later use.

2. Query-subsystem: a fingerprint query image is received as input (arrow 1). Once, the fingerprint ROI is detected (module A, arrow 2) the feature extraction algorithms contained in the descriptor library are used to extract the feature vectors from the query image (module B with arrows 3 and 4, respectively). The query image feature vector is used to rank the database images according to their similarity to the query image (module $\mathrm{C}$ ). For that purposes, a distance computation algorithm is first selected from the descriptor library (arrow 5), and a subset of feature vectors are retrieved from the database by considering their distance to the query image feature vector (arrow 6). Finally, the most similar database images are ranked (arrow 7) and returned to the user (arrow 8).

\section{Wavelet-Based Feature Extraction}

In our approach, three main steps are needed to compute the feature vectors: (1) construct a circular tesellation around a reference point, (2) decompose the fingerprint images into a set of scale and orientation tunnable components, (3) compute the average absolute deviation $(A A D)$ from the tesellated sectors of the decomposed images.

\subsection{Fingerprint Image Tesellation}

To compute the multi-resolution and multi-orientation feature vector, a region of interest (ROI) represented by a circular tesellation around a reference point is used. Let $I(x, y)$ be the gray level image value at pixel $(x, y)$ of a fingerprint of size $M \times N$, and $\left(x_{r}, y_{r}\right)$ be the location of the reference point, the circular tesellation of radius $r$ is composed by a set of independent sectors $S_{i, j}$ at different bands $j$ defined as [7]:

$$
\begin{aligned}
S_{i, j}= & \left\{(x, y) \mid(i-1) \cdot b+b_{o} \leq r<i \cdot b+b_{o}\right. \\
& \left.\theta_{j} \leq \theta<\theta_{j+1}, 1 \leq x \leq M, 1 \leq y \leq N\right\}
\end{aligned}
$$


where $\theta_{j}=(i \bmod k) \cdot(2 \pi / k), b$ denotes the bandwidth and $b_{o}$ is the width of the most inner band around the reference point, and $j=0 \ldots B \times k-1$, where $B$, and $k$ represent the number of cocentric bands, and the number of sectors in each band, respectively. Considering that the images we used for test purposes were scanned at 500 dpi, we considered $B=5$ bands, each of 20 pixels wide, and a total number of $k=16$ sectors.

\subsection{Fingerprint Image Decomposition}

We exploit the capability of two effective bandpass multiresolution techniques for capturing useful fingerprint information at different scales and orientations. These techniques, include Gabor-, and Steerable-Wavelets [8,9]. Moreover, the use of these image decomposition approaches is motivated by the following reasons: (1) they integrate both multiresolution, and space-frequency properties naturally, therefore both global and local fingerprint information can be captured, (2) their tunable components make them flexible to different computation and recognition needs, and finally (3) they have demonstrated high accuracy in texture analysis applications [8]. The fingerprint $R O I$ is decomposed into a set of orientation, and scale components by using the mentioned image transforms.

\subsection{Feature Vector Extraction}

To generate feature vectors, statistical measures are applied. More precisely, we use the average absolute deviation of the mean value at each sector of the decomposed images. The feature value $F_{i \theta s}$ is computed as follows [7]:

$$
F_{i \theta s}=\frac{1}{K_{i \theta s}}\left(\sum_{(x, y) \in S_{i \theta s}}\left|S_{i \theta s}(x, y)-S_{i \theta s}^{-}(x, y)\right|\right)
$$

where $S_{i \theta s}$ represents the sector at position $i$ in the decomposed image at orientation $\theta$, and at scale $s . K_{i \theta s}$ is the number of pixels in sector $S_{i \theta s}$, and $S_{i \theta s}^{-}$represents the mean value of pixels in sector $S_{i \theta s}$. The feature vectors of an input fingerprint image are represented in Figure 2 by using tesellated images with five bands and sixteen sectors.

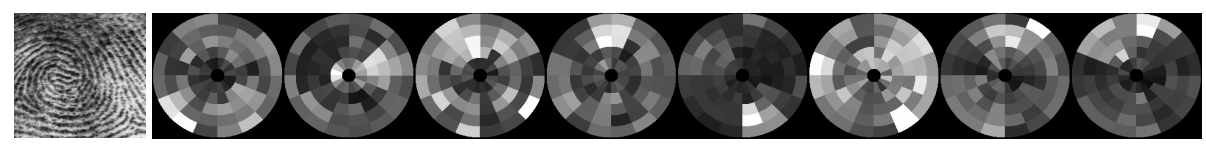

Fig. 2. Feature vector representation of a fingerprint image

\section{Fingerprint Feature Classification}

This section aims to present the new approach for pattern recognition called OPF (Optimum-Path Forest). It demonstrated to be generally more efficient than Artificial Neural Networks and Support Vector Machines in some applications [6]. The OPF approach works by modeling the patterns as being nodes of a graph in the feature space, 
where every pair of nodes is connected by an arc (complete graph). This classifier creates a discrete optimal partition of the feature space such that any unknown sample can be classified according to this partition. This partition is an optimum path forest computed in $\Re^{n}$ by the image foresting transform (IFT) algorithm [10].

Let $Z_{1}$ and $Z_{2}$ be training and test sets with $\left|Z_{1}\right|$ and $\left|Z_{2}\right|$ samples such as feature vectors. Let $\lambda(s)$ be the function that assigns the correct label $i, i=1,2, \ldots, c$, from class $i$ to any sample $s \in Z_{1} \cup Z_{2}$. Let $S \subset Z_{1}$ be a set of prototypes of all classes (i.e., key samples that best represent the classes). Let $v$ be an algorithm which extracts $n$ attributes (texture properties) from any sample $s \in Z_{1} \cup Z_{2}$ and returns a vector $\boldsymbol{v}(s) \in \Re^{n}$. The distance $d(s, t)$ between two samples, $s$ and $t$, is the one between their feature vectors $\boldsymbol{v}(s)$ and $\boldsymbol{v}(t)$ (Figure 3 h) (e.g., Euclidean or any valid metric).

Let $\left(Z_{1}, A\right)$ be a complete graph whose the nodes are the samples in $Z_{1}$. We define a path as being a sequence of distinct samples $\pi=\left\langle s_{1}, s_{2}, \ldots, s_{k}\right\rangle$, where $\left(s_{i}, s_{i+1}\right) \in A$ for $1 \leq i \leq k-1$. A path is said trivial if $\pi=\left\langle s_{1}\right\rangle$. We assign to each path $\pi$ a cost $f(\pi)$ given by a path-cost function $f$. A path $\pi$ is said optimum if $f(\pi) \leq f\left(\pi^{\prime}\right)$ for any other path $\pi^{\prime}$, where $\pi$ and $\pi^{\prime}$ end at a same sample $s_{k}$. We also denote by $\pi \cdot\langle s, t\rangle$ the concatenation of a path $\pi$ with terminus at $s$ and an arc $(s, t)$. The OPF algorithm uses the path-cost function $f_{\max }$, because of its theoretical properties for estimating optimum prototypes:

$$
\begin{aligned}
f_{\max }(\langle s\rangle) & = \begin{cases}0 & \text { if } s \in S, \\
+\infty & \text { otherwise }\end{cases} \\
f_{\max }(\pi \cdot\langle s, t\rangle) & =\max \left\{f_{\max }(\pi), d(s, t)\right\}
\end{aligned}
$$

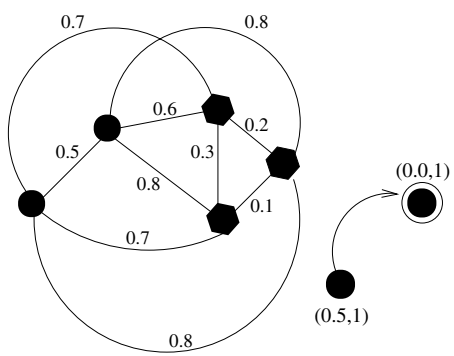

(a)

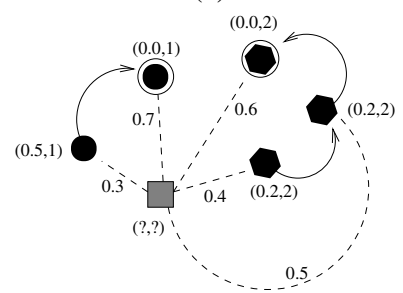

(c)

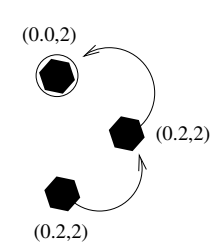

(b)

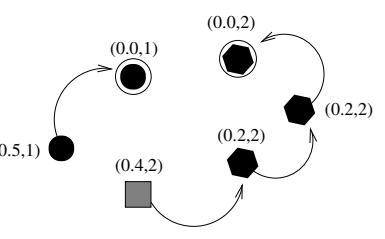

(d)

Fig. 3. (a) Complete weighted graph for a simple training set. (b) Resulting optimum-path forest for $f_{\max }$ and two given prototypes (circled nodes). The entries $(x, y)$ over the nodes are, respectively, the cost and the label of the samples. The directed arcs indicate the predecessor nodes in the optimum path. (c) Test sample (gray square) and its connections (dashed lines) with the training nodes. (d) The optimum path from the most strongly connected prototype, its label 2, and classification cost 0.4 are assigned to the test sample. 
We can observe that $f_{\max }(\pi)$ computes the maximum distance between adjacent samples in $\pi$, when $\pi$ is not a trivial path.

The OPF algorithm assigns one optimum path $P^{*}(s)$ from $S$ to every sample $s \in Z_{1}$, forming an optimum path forest $P$ (a function with no cycles which assigns to each $s \in Z_{1} \backslash S$ its predecessor $P(s)$ in $P^{*}(s)$ or a marker nil when $s \in S$ (Figure 3b). Let $R(s) \in S$ be the root of $P^{*}(s)$ which can be reached from $P(s)$. The OPF algorithm computes for each $s \in Z_{1}$, the cost $C(s)$ of $P^{*}(s)$, the label $L(s)=\lambda(R(s))$, and the predecessor $P(s)$, as follows.

\section{Algorithm 1 - OPF ALGORITHM}

INPUT: $\quad \mathrm{A} \lambda$-labeled training set $Z_{1}$, prototypes $S \subset Z_{1}$ and the pair $(v, d)$ for feature vector and distance computations.

Output: $\quad$ Optimum path forest $P$, cost map $C$ and label map $L$.

AUXILIARY: Priority queue $Q$ and cost variable $c s t$.

1. For each $s \in Z_{1} \backslash S$, set $C(s) \leftarrow+\infty$.

2. For each $s \in S$, do

3. $\mathrm{L} C(s) \leftarrow 0, P(s) \leftarrow n i l, L(s) \leftarrow \lambda(s)$, and insert $s$ in $Q$.

4. While $Q$ is not empty, do

5. $\quad$ Remove from $Q$ a sample s such that $C(s)$ is minimum.

6. $\quad$ For each $t \in Z_{1}$ such that $t \neq s$ and $C(t)>C(s)$, do

7. $\quad$ Compute cst $\leftarrow \max \{C(s), d(s, t)\}$.

8. $\quad$ If cst $<C(t)$, then

9. $\quad$ If $t \in Q$, then remove t from $Q$.

10. $L[P(t) \leftarrow s, L(t) \leftarrow L(s), C(t) \leftarrow$ cst, and insert $t$ in $Q$.

Lines $1-3$ initialize maps and insert prototypes in $Q$. The main loop computes an optimum path from $S$ to every sample $s$ in a non-decreasing order of cost (Lines 4-10). At each iteration, a path of minimum $\operatorname{cost} C(s)$ is obtained in $P$ when we remove its last node $s$ from $Q$ (Line 5). Lines $8-10$ evaluate if the path that reaches an adjacent node $t$ through $s$ is cheaper than the current path with terminus $t$ and update the position of $t$ in $Q, C(t), L(t)$ and $P(t)$ accordingly. The label $L(s)$ may be different from $\lambda(s)$, leading to classification errors in $Z_{1}$. The training finds prototypes with minimum classification errors in $Z_{1}$. The OPF algorithm works with two phases: training and classification (test), as follows. The prototypes set $\mathrm{S}$ are found in the training phase, as described below.

\subsection{Training Phase}

We say that $S^{*}$ is an optimum set of prototypes when Algorithm 1 minimizes the classification errors for every $s \in Z_{1}$. Set $S^{*}$ can be found by exploiting the theoretical relation between Minimum Spanning Tree (MST) [11] and optimum path tree for $f_{\text {max }}$. The training essentially consists of finding $S^{*}$ and an OPF classifier rooted at $S^{*}$.

By computing an MST in the complete graph $\left(Z_{1}, A\right)$, we obtain a connected acyclic graph whose nodes are all samples in $Z_{1}$ and the arcs are undirected and weighted by the distance $d$ between the adjacent sample feature vectors. This spanning tree is optimum in the sense that the sum of its arc weights is minimum as compared to any other 
spanning tree in the complete graph. In the MST, every pair of samples is connected by a single path which is optimum according to $f_{\max }$. That is, for any given sample $s \in Z_{1}$, it is possible to direct the arcs of the MST such that the result will be an optimum path tree $P$ for $f_{\max }$ rooted at $s$. The optimum prototypes are the closest elements in the MST with different labels in $Z_{1}$. By removing the arcs between different classes, their adjacent samples become prototypes in $S^{*}$ and Algorithm 1 can compute an optimum-path forest with high accuracy in $Z_{1}[12]$.

On the other side, we are currently working with different approaches to choose the prototypes set [13], leading to different accuracies, depending on the application. In all of these approaches, one interesting point is that the number of prototypes encodes how much a feature space is overlapped. If we have a high number of prototypes, this means that, for MST approach, for instance, we have a lot of samples from different classes in the decision region, leading to a high number of misclassifications in the test phase, or even so in the training phase for other classifiers. In the OPF version presente here, a high number of these problematic samples will be prototypes, and can not be misclassified.

\subsection{Classification}

For any sample $t \in Z_{2}$, the OPF consider all arcs connecting $t$ with samples $s \in Z_{1}$, as though $t$ were part of the graph (Figure 3r). Considering all possible paths from $S^{*}$ to $t$, we wish to find the optimum path $P^{*}(t)$ from $S^{*}$ and label $t$ with the class $\lambda(R(t))$ of its most strongly connected prototype $R(t) \in S^{*}$ (Figure 3 d). This path can be identified incrementally, by evaluating the optimum cost $C(t)$ as

$$
C(t)=\min \{\max \{C(s), d(s, t)\}\}, \forall s \in Z_{1} .
$$

Let the node $s^{*} \in Z_{1}$ be the one that satisfies the above equation (i.e., the predecessor $P(t)$ in the optimum path $\left.P^{*}(t)\right)$. Given that $L\left(s^{*}\right)=\lambda(R(t))$, the classification simply assigns $L\left(s^{*}\right)$ as the class of $t$. An error occurs when $L\left(s^{*}\right) \neq \lambda(t)$.

\section{Experimental Setup}

To test our approach, we used the NIST special database 4 (NIST-4) [14]. It comprises 2000 pairs of fingerprint images. The size of each image is $480 \times 512$ pixels with a resolution of 500 DPI. Each fingerprint is assigned into one of the following five classes: Whorl (W), Right Loop (R), Left Loop (L), Arch (A), and Tented Arch (T). To resemble a real distribution of fingerprint classes, the cardinality of the five classes was adapted, leading to a database of 1024 pairs of images $(\mathrm{W}=27.9 \%$, $\mathrm{R}=31.7 \%, \mathrm{~L}=33.8 \%, \mathrm{~A}=3.7 \%, \mathrm{~T}=2.9 \%$ ). The first fingerprint instances are used as image databases, whereas the second ones as query fingerprints.

\section{Experimental Results}

To demonstrate the discriminating power of our classification methods, we conducted two series of experiments. In the first series of experiments (Subsection 6.1), we evaluated the recognition accuracy of the proposed exclusive classification approach, whilst 
the second series of experiments (Subsection 6.2), are used to evaluate the effectiveness of the continuous classification method.

In both series of experiments, we used different multi-resolution parameters for the Gabor and Steerable Decomposition methods. Our experiments agree with [15] in that, the most relevant textural information in images is contained in the first two levels of decomposition, since little recognition improvement is achieved by varying the number of scales during image decomposition. Therefore, we focus our discussions on image decompositions having $(S=2)$ scales at several orientations $(K=4,5,6,7,8)$.

The dimensionality of the feature vectors depends on four parameters: the number of scales $(S)$ and the number of orientations $(O)$ considered during multi-resolution image decomposition, as well as the number of bands $(B)$ and sectors $(k)$ during fingerprint image tesellation. Thus, the total length of the feature vectors is computed as follows: $O \times S \times B \times k$. An important motivation in our study was to study the impact of different multi-resolution settings in the recognition accuracies of our approaches. By doing this, different computational needs may be attended.

\subsection{Exclusive Classification Approach}

In our experiments, the accuracy is measured by taking into account that the classes may have different sizes in $Z_{2}$ [6]. In all experiments, the NIST-4 dataset was divided into two parts: a training set $Z_{1}$ with $50 \%$ of the samples and a test set $Z_{2}$ with also $50 \%$ of the samples. These samples were randomly selected and each experiment was repeated 10 times with different sets $Z_{1}$ and $Z_{2}$ to compute the mean accuracy.

The accuracy of our exclusive classification approach is summarized in Table 1, It compares the recognition accuracy obtained by the Gabor and Steerable Wavelets using ( $S=2$ ) scales with different orientations $(K=4,5,6,7,8)$. Furthermore, for each different pair of parameter combinations, we also computed the recognition accuracy using the following similarity measures: Bray Curtis, Canberra, Euclidean, Manhattan, Square Chord, and Square Chi-Squared distances. The referred Table reveals some relevant information:

1. First, by comparing the recognition accuracies of the Gabor Wavelets and the Steerable Wavelets, one can observe that regardless of the parameter settings, the Steerable Wavelets achieve higher recognition rates.

2. Second, for the different Gabor and Steerable decompositions, the higher recognition accuracies were achieved by using either the Euclidean or the Manhattan distances. This observation is relevant since it allow us, on the one hand, to demonstrate the stability of our image descriptors across the different parameter configurations, on the other, it help us to decide which similarity measure will perform better in most of the cases.

3. Finally, higher recognition rates are obtained by using more number of orientations. These results make intuitive sense since by using more orientations, more information is captured at different angles. However, as one can see, the difference of recognition rates across the different orientations is slight.

By considering the achieved classification rates described in Table 1, we selected the image descriptor with highest recognition rates to display the resultant confusion matrix 
Table 1. Classification accuracy summarization using the OPF classifier obtained in the NIST4 database using $(S=2)$ scales with $(K=4,5,6,7,8)$ orientations for Gabor and Steerable Wavelets

\begin{tabular}{|c||c|c||c|c||c|c||c|c||c||c|}
\hline \multirow{2}{*}{ Metric } & \multicolumn{2}{|c|}{$S=2, K=4$} & \multicolumn{2}{c|}{$S=2, K=5$} & \multicolumn{2}{c|}{$S=2, K=6$} & \multicolumn{2}{|c|}{$S=2, K=7$} & \multicolumn{2}{c|}{$S=2, K=8$} \\
\cline { 2 - 10 } & Gabor & Steer & Gabor & Steer & Gabor & Steer & Gabor & Steer & Gabor & Steer \\
\hline \hline Bray Curtis & 85.83 & $\mathbf{8 6 . 7 3}$ & 86.13 & $\mathbf{8 6 . 9 9}$ & 86.57 & $\mathbf{8 7 . 3 4}$ & 86.31 & $\mathbf{8 7 . 0 9}$ & 86.01 & $\mathbf{8 6 . 9 8}$ \\
\hline Canberra & 86.51 & $\mathbf{8 7 . 1 4}$ & 86.30 & $\mathbf{8 7 . 1 0}$ & 85.83 & $\mathbf{8 6 . 8 2}$ & 85.68 & $\mathbf{8 7 . 0 4}$ & 85.21 & $\mathbf{8 6 . 7 1}$ \\
\hline Euclidean & 85.45 & $\mathbf{8 8 . 6 1}$ & 86.92 & $\mathbf{8 8 . 0 3}$ & 86.19 & $\mathbf{8 8 . 7 6}$ & 86.17 & $\mathbf{8 8 . 5 1}$ & 86.07 & $\mathbf{8 9 . 6 4}$ \\
\hline Manhattan & 85.58 & $\mathbf{8 7 . 5 6}$ & 87.19 & $\mathbf{8 8 . 2 0}$ & 85.96 & $\mathbf{8 8 . 8 9}$ & 87.44 & $\mathbf{8 9 . 2 9}$ & 86.68 & $\mathbf{8 9 . 4 0}$ \\
\hline Square Chord & 85.80 & $\mathbf{8 7 . 6 4}$ & 85.61 & $\mathbf{8 7 . 5 2}$ & 85.28 & $\mathbf{8 8 . 8 1}$ & 85.28 & $\mathbf{8 7 . 6 2}$ & 84.44 & $\mathbf{8 7 . 6 9}$ \\
\hline Square Chi-Squared & 85.42 & $\mathbf{8 6 . 9 2}$ & 84.89 & $\mathbf{8 8 . 0 9}$ & 85.08 & $\mathbf{8 7 . 9 1}$ & 84.85 & $\mathbf{8 8 . 3 9}$ & 84.85 & $\mathbf{8 8 . 6 5}$ \\
\hline
\end{tabular}

Table 2. (a) Confusion matrix for Steerable Wavelets $(S=2, K=8)$ with Euclidean distance. (b) Confusion matrix for Gabor Wavelets $(S=2, K=8)$ with Euclidean distance. (c) Classification results summarization of our approach against the method of Yao et al [16].

(a)

\begin{tabular}{|c|c|c|c|c|c|}
\hline & $\mathrm{W}$ & $\mathrm{R}$ & $\mathrm{A}$ & $\mathrm{L}$ & $\mathrm{T}$ \\
\hline \hline $\mathrm{W}$ & $\mathbf{2 5 0}$ & 10 & 0 & 26 & 0 \\
\hline $\mathrm{R}$ & 16 & $\mathbf{2 9 3}$ & 6 & 4 & 6 \\
\hline $\mathrm{A}$ & 0 & 4 & $\mathbf{2 9}$ & 0 & 4 \\
\hline $\mathrm{L}$ & 8 & 18 & 4 & $\mathbf{3 1 5}$ & 2 \\
\hline $\mathrm{T}$ & 2 & 6 & 2 & 0 & $\mathbf{1 9}$ \\
\hline
\end{tabular}

(b)

\begin{tabular}{|c|c|c|c|c|c|}
\hline & $\mathrm{W}$ & $\mathrm{R}$ & $\mathrm{A}$ & $\mathrm{L}$ & $\mathrm{T}$ \\
\hline $\mathrm{W}$ & $\mathbf{2 4 2}$ & 16 & 0 & 26 & 2 \\
\hline $\mathrm{R}$ & 0 & $\mathbf{3 0 1}$ & 2 & 16 & 6 \\
\hline $\mathrm{A}$ & 0 & 2 & $\mathbf{2 1}$ & 14 & 0 \\
\hline $\mathrm{L}$ & 2 & 22 & 4 & $\mathbf{3 1 5}$ & 4 \\
\hline $\mathrm{T}$ & 0 & 4 & 2 & 8 & $\mathbf{1 5}$ \\
\hline
\end{tabular}

(c)

\begin{tabular}{|c|c|}
\hline Method & Accuracy \\
\hline RNN [16] & $71.5 \%$ \\
\hline SVM [16] & $89.1 \%$ \\
\hline SVM+RNN [16] & $90.0 \%$ \\
\hline Our approach & $89.64 \%$ \\
\hline
\end{tabular}

for the five fingerprint classes. This image descriptor corresponds to the combination of Steerable Wavelets for feature extraction with the Euclidean distance for similarity measurement. Tables 2(a) and 2(b) show how Steerable Wavelets outperform Gabor Wavelets on the five fingerprint classes.

We also compared the accuracy of our method against an approach that uses texture information for fingerprint characterization and combines two well known approaches to increase fingerprint classification rates, those approaches are namely support vector machines (SVM) and recursive neural networks (RNN) [16] (See Table 2(c)). From the results, we can see that our method obtains high classification rates without the need of combining two classifiers for achieving higher classification rates 1

\subsection{Continuous Classification Analysis}

The accuracy of our continuous classification approach was evaluated by using the retrieval-accuracy vs. penetration-rate curves [17]. The retrieval-accuracy is defined as the average number of correctly retrieved fingerprints, whereas the penetration-rate represents the number of images in the database. The search is said to be successful, if one of the retrieved images belongs to the same finger as the query.

\footnotetext{
${ }^{1}$ Note that, the OPF classifier has proven to be at least 10 times faster than the SVM classifier in most of the cases [6].
} 


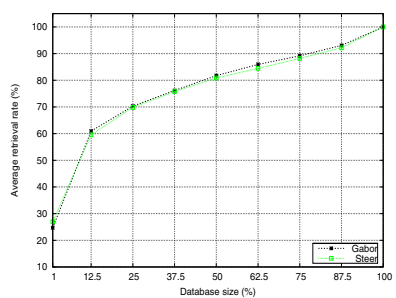

(a)

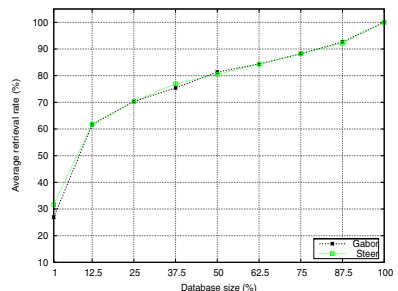

(d)

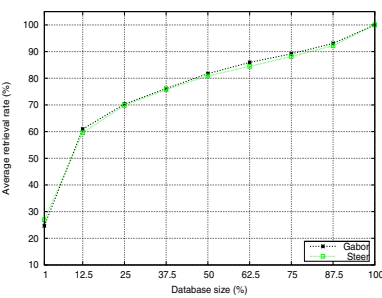

(b)

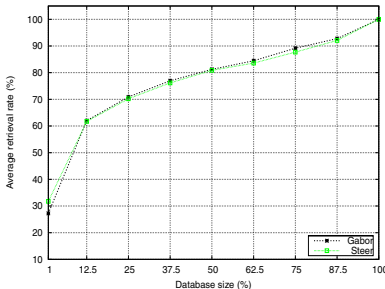

(e)

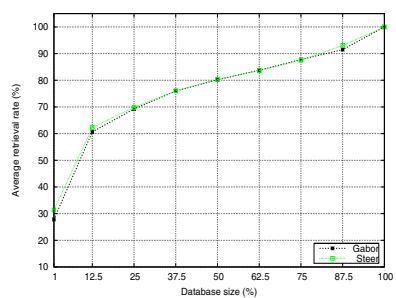

(c).

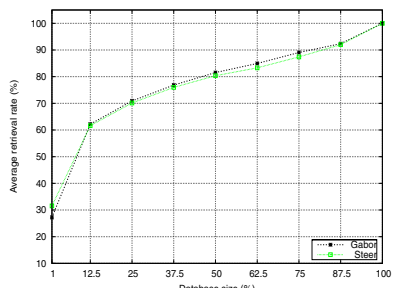

(f)

Fig. 4. Effectiveness of our various image descriptors. Each subfigure represents the higher retrieval accuracies obtained for each different similarity measure. Average retrieval rates using: (a) Bray Curtis distance. (b) Canberra distance. (c) Euclidean distance. (d) Manhattan distance. (e) Square Chi-Squared distance. (f) Square Chord distance.

In these series of experiments, we wanted to know how well our image descriptors performed in the context of continuous classification schemes, e.g., no a priori information is given about the fingerprint classes, and thus no classifier should be used for recognition purposes. The fingerprints are just represented by feature vectors spread over a feature space. Ideally, feature vectors corresponding to related fingerprints will be close to each other. The accuracy of our continuous classsification approach is ilustrated in Figure 4. Since an image descriptor consists of a pair of feature extraction method with a similarity measure, we show the best combination of similarity measure with parameter settings for both Gabor and Steerable Wavelets.

In our experiments, we varied the size of the fingerprint database by considering different numbers of top retrieved images ( $x$-axis). For each different database size, we computed the relevant retrieval average of both feature extraction methods $(y$-axis). In the ideal case, a perfect retrieval accuracy is said to be achieved, if the average retrieval rate is equal to $100 \%$ by considering just $1 \%$ of the database size.

From the set of Figures, one can see that both feature extraction methods performs almost the same. However, Steerable Wavelets perform better than Gabor Wavelets for almost each different similarity measure. As in the exclusive classification approach, the higher accuracies were achieved by using the Euclidean distance. This means that our image descriptors perform well for both exclusive and continuous applications. Furthermore, as it can be noticed, by just using $12.5 \%$ of the images during the retrieval phase, our descriptors are able to identificate more than $60 \%$ of the fingerprint images. At the same time, an accuracy of almost $80 \%$ is achieved by considering just $37.5 \%$ of the database size. These results are very promising and demonstrate the effectiveness of our approach. 


\section{Conclusions}

In this paper a new scheme for fingerprint classification was proposed, that is suitable for both exclusive and continuous classification domains. Its main features include: (1) a set of texture-based image descriptors composed by feature extraction methods and similariy measures, and (2) a novel multi-class recognition method based on Optimum Path Forest.

The feature representation methods exploit the discriminability properties of two multi-resolution approaches to capture relevant fingerprint texture information. Furthermore, an importat key characteristic of our representation methods is that they enconde both global and local fingerprint information into a single feature vector. The global information of fingerprint images is captured by a set of image representations located at different scales and orientations, whilst the local information is captured in each decomposed image by a tesellation grid. By doing this, reliable and discriminant fingerprint characteristics are captured. Since similarity measurement for feature representations plays also an important role in the recognition rates, we evaluated how different combinations of feature representations with similarity measures affect the classification rates. From our results, we conclude that our feature representation methods are benefited by using the Euclidean or Manhattan distances for similarity measurement purposes.

In addition, our system adopted a novel approach for pattern classification based on Optimum Path Forest, which finds prototypes with minimum classification errors in the training set. By combining the discriminating power of our image descriptors and classifier, our approaches achieved high classification rates.

\section{Acknowledgments}

This work was partially supported by CAPES, FAPESP, CNPQ, and Microsoft Research.

\section{References}

1. Pankanti, S., Prabhakar, S., Jain, A.K.: On the individuality of fingerprints. IEEE Transactions on Pattern Analysus and Machine Intelligence 24, 1010-1025 (2002)

2. Jain, A.K., Ross, A., Pankanti, S.: Biometrics: A tool for information security. IEEE Transactions on Information Forensics and Security 1, 125-143 (2006)

3. Jain, A.K., Chen, Y., Demirkus, M.: Pores and ridges: High-resolution fingerprint matching using level 3 features. IEEE Transactions on PAMI 29, 15-27 (2007)

4. Yager, N., Amin, A.: Fingerprint classification: a review. Pattern Anal. Appl. 7, 77-93 (2004)

5. Lumini, A., Maio, D., Maltoni, D.: Continuous versus exclusive classification for fingerprint retrieval. Pattern Recognition Letters 18, 1027-1034 (1997)

6. Papa, J., Falcão, A., Suzuki, C., Mascarenhas, N.: A discrete approach for supervised pattern recognition. In: Brimkov, V.E., Barneva, R.P., Hauptman, H.A. (eds.) IWCIA 2008. LNCS, vol. 4958, pp. 136-147. Springer, Heidelberg (2008)

7. Jain, A.K., Prabhakar, S., Hong, L., Pankanti, S.: Filterbank-based fingerprint matching. IEEE Transactions on Image Processing 9, 846-859 (2000)

8. Manjunath, B.S., Ma, W.Y.: Texture features for browsing and retrieval of image data. IEEE Transactions on Pattern Analysis and Machine Intelligence 18, 837-842 (1996) 
9. Portilla, J., Simoncelli, E.P.: A parametric texture model based on joint statistics of complex wavelet coefficients. International Journal of Computer Vision 40, 49-70 (2000)

10. Falcão, A., Stolfi, J., Lotufo, R.: The image foresting transform: theory, algorithms, and applications. IEEE Trans. Pattern Anal. Mach. Intell. 26, 19-29 (2004)

11. Cormen, T., Leiserson, C., Rivest, R.: Introduction to Algorithms. MIT Press, Cambridge (1990)

12. Cousty, J., Bertrand, G., Najman, L., Couprie, M.: Watersheds, minimum spanning forests, and the drop of water principle, École Supérieure d'Ingénieurs (2007)

13. Papa, J., Falcão, A.: A new variant of the optimum-path forest classifier. In: 4th International Symposium on Visual Computing (accepted, 2008)

14. Watson, C., Wilson, C.: Nist special database 4, fingerprint database. U.S. National Institute of Standards and Technology (1992)

15. Do, M.N., Vetterli, M.: Wavelet-based texture retrieval using generalized gaussian density and kullback-leibler distance. IEEE Transactions on Image Processing 11, 146-158 (2002)

16. Yao, Y., Marcialis, G.L., Pontil, M., Frasconi, P., Roli, F.: Combining flat and structured representations for fingerprint classification with recursive neural networks and support vector machines. Pattern Recognition 36, 397-406 (2003)

17. Cappelli, R., Lumini, A., Maio, D., Maltoni, D.: Fingerprint classification by directional image partitioning. IEEE Trans. Pattern Anal. Mach. Intell. 21, 402-421 (1999) 